\title{
Um Sistema Multiagente no Auxílio à Formação de Grupos de Aprendizagem Colaborativa
}

\author{
Cícero C. Quarto ${ }^{1}$, Reinaldo de J. da Silva ${ }^{1}$, Nécio de Lima Veras ${ }^{2}$, Edson \\ Nascimento $^{3}$, Claudio S. Hutz ${ }^{4}$, Magda Bercht ${ }^{5}$ \\ ${ }^{1}$ Centro de Ciências Tecnológicas (UEMA) - São Luís - MA - Brazil \\ ${ }^{2}$ Departamento de Ciência da Computação (IFCE) - Tianguá - Ceará - Brazil \\ ${ }^{3}$ Biological Information Processing (UFMA) - São Luís - MA - Brazil \\ ${ }^{4}$ Instituto de Psicologia (UFRGS) - Porto Alegre - RS - Brazil \\ 5Instituto de Informática (UFRGS) - Porto Alegre - RS - Brazil \\ \{cicero, reinaldo\}@engcomp.uema.br,necio.veras@ifce.edu.br, \\ nascimento.edson02@gmail.com, claudio.hutz@gmail.com, \\ bercht@inf.ufrgs.br
}

\begin{abstract}
This paper presents a multi-agent system to aid in the formation of collaborative learning groups. Collaboration leads to positive results when group members build knowledge together, acquire skills, resolve conflicts and articulate with each other's interpersonal skills. Group formation without carefully chosen criteria often causes problems such as the disproportional distribution of individuals as well as lack of motivation and growing resistance towards group work. The proposed technological model has been designed using Prometheus methodology and JaCaMo framework. The obtained experimental results are promising, especially regarding the formation of higher performance groups in collaborative learning contexts.
\end{abstract}

Resumo. Este trabalho apresenta um sistema multiagente no apoio à formação de grupos de aprendizagem colaborativa. A colaboração leva a resultados positivos quando os membros do grupo constroem conhecimentos juntos, adquirem habilidades, resolvem conflitos e articulam entre si habilidades interpessoais. Formar grupos sem critérios cuidadosos muitas vezes provoca problemas como a participação desproporcional de indivíduos, desmotivação e resistência ao trabalho de grupo. O modelo de tecnologia proposto foi concebido utilizando a metodologia Prometheus e o framework JaCaMo. Os resultados obtidos nos experimentos são promissores rumo a composições de grupos de alta performance para contextos de aprendizagem colaborativa. 
V Congresso Brasileiro de Informática na Educação (CBIE 2016)

Anais do XXVII Simpósio Brasileiro de Informática na Educação (SBIE 2016)

\section{Introdução}

Pesquisas vêm focando na determinação de parâmetros que podem afetar a aprendizagem colaborativa e como eles podem ser considerados na tecnologia educacional [Stahl et al. 2006]; [Monteserin et al. 2010]; [Reis et al. 2015a]. Para [So and Brush 2008], pesquisas na literatura destacam problemas relacionados à resistência e desmotivação dos alunos para trabalhar em grupo. Isto está associado à percepção dos alunos sobre o quanto eles pertencem ao grupo e estão conectados aos demais colegas, influenciada, ao mesmo tempo, pela ligação afetiva entre os alunos no trabalho em grupo, sendo este elemento visto como um dos fatores críticos que afetam a motivação dos alunos e o engajamento na colaboração [So and Brush 2008].

Este trabalho apresenta um sistema multiagente no apoio à formação otimizada de grupos a partir da autoavaliação de comportamento psicológico (perfis de autorrelatos) de alunos, de forma que os benefícios da aprendizagem colaborativa sejam alcançados de forma plena.

O trabalho é estruturado em mais cinco seções, a saber: A Seção 2, apresenta conceitos que apoiam a pesquisa em Psicologia Positiva. A Seção 3 descreve o sistema multiagente. A metodologia de pesquisa é descrita na Seção 4. A Seção 5 é reservada para as análises e discussões de resultados. As Conclusões e trabalhos futuros são apresentados na Seção 6.

\section{Psicologia Positiva}

A Psicologia Positiva foi inicialmente definida como uma Ciência da experiência positiva subjetiva, traços individuais positivos e instituições positivas que procura melhorar a qualidade de vida dos indivíduos [Seligman and Csikszentmihalyi 2000]. Dos estudos de [Tierney 1995]; [Seligman and Csikszentmihalyi 2000]; [Snyder et al. 2002]; [Baumeister et al. 2003]; [Bressler et al. 2011] e [Pacico and Bastianello 2014], pode-se constatar que as pesquisas em psicologia positiva enfatizam uma atenção às variáveis psicológicas positivas esperança, autoestima e autoeficácia no impacto destas no desempenho educacional do indivíduo. Portanto, motivado por este contexto, assim como os objetivos deste trabalho, considera-se e investiga-se a influência destas variáveis na formação otimizada de grupos para potencializar a aprendizagem colaborativa.

[Hanson et al. 2010]; [Bressler et al. 2011] definem esperança como sendo cognições voltadas para a obtenção de um objetivo, compostas por rotas e agenciamento. $\mathrm{O}$ agenciamento é a motivação do sujeito em perseguir a meta a ser alcançada, e as rotas são os caminhos planejados para obter tais objetivos. Em [Baumeister et al. 2003]; [Hutz et al. 2014], tem-se que a autoestima representa um aspecto avaliativo do autoconceito e consiste em um conjunto de pensamentos referentes a si mesmo. Tratase, portanto, de uma orientação positiva (autoaprovação) ou negativa (depreciação) de se voltar para si próprio e, nessa concepção, ela é a representação pessoal dos sentimentos gerais e comuns de autovalor. Para [Bandura 1997], a autoeficácia é definida como a crença na capacidade de realizar uma determinada tarefa, com base nos próprios recursos. Em [Hutz et al. 2014], tem-se que escalas de autorrelatos são instrumentos de avaliação para coleta de informações, às quais auxiliam a conhecer e entender melhor o indivíduo e conclui que estas são uma forma válida para avaliar afetos. Para as mensurações dos perfis de autorrelatos considerados neste trabalho, utilizou-se as 
V Congresso Brasileiro de Informática na Educação (CBIE 2016)

Anais do XXVII Simpósio Brasileiro de Informática na Educação (SBIE 2016)

escalas de Esperança Disposicional [Pacico and Bastianello 2014], Escala de Autoeficácia [Pacico et al. 2014] e a Escala de Autoestima [Hutz et al. 2014]. Na Tabela 1 são mostrados cinco níveis de perfis de autorrelatos do indivíduo em função das faixas de percentis, estabelecidos nas normas de cada escala supracitada.

Tabela 1: Perfis de autorrelatos Vs. Percentis.

Fonte: Laboratório de Mensuração do Inst. Psicologia da UFRGS, 2015.

\begin{tabular}{c|c}
\hline Faixas de Percentil & Níveis de Autorrelatos dos alunos \\
\hline$<30 \%$ & Baixo (B) \\
\hline$\geq 30 \% \mathrm{e}<40 \%$ & Médio Baixo (MB) \\
\hline$\geq 40 \% \mathrm{e}<60 \%$ & Médio (M) \\
$\geq 60 \% \mathrm{e}<70 \%$ & Médio Alto (MA) \\
\hline$\geq 70 \%$ & Alto (A) \\
\hline
\end{tabular}

\section{O Sistema Multiagente Hermes}

O uso da tecnologia de agentes de software se apresenta como uma estratégia promissora para ser aplicada aos desafios dos ambientes educacionais modernos que estão cada vez mais influenciados por tecnologias como Internet e Inteligência Artificial (IA) [Cunha et al. 2002]. Em [Wooldridge 2008]; [Russell and Norvig 2013], tem-se que um agente é uma entidade capaz de perceber um ambiente através de sensores e atuar sobre o mesmo por meio de atuadores. Conforme [Weiss 1999], a Inteligência Artificial Distribuída (IAD) é estudo, construção e aplicação de sistemas multiagentes (SMA), ou seja, sistemas baseados em agentes, onde estes almejam realizar um conjunto de tarefas. Seguindo [Wooldridge 2008]; [Russel and Norvig 2013] e aliando aos aspectos descritos na Seção 2, a abordagem foi materializada em um modelo de sistema baseado na tecnologia de agentes, denominado SMA-Hermes. A Figura 1 ilustra a arquitetura do modelo e logo em seguida são descritos seus componentes.

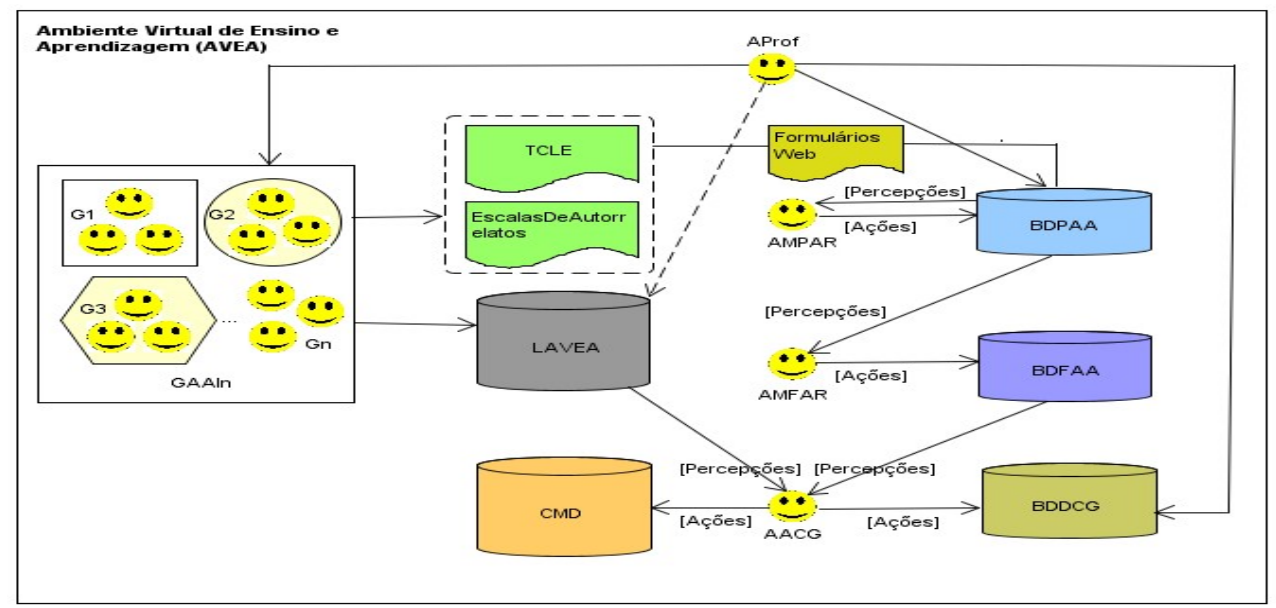

Figura 1: Arquitetura geral do SMA-Hermes.

Fonte: Autor (2016).

Grupos de Agentes Alunos - o Grupo de Agentes Alunos (GAAln) representa grupos de alunos no Ambiente Virtual de Ensino e Aprendizagem (AVEA), realizando suas atividades de aprendizagem dentro de uma perspectiva colaborativa, que buscam compartilharem suas habilidades sociais e cognitivas, decisões tomadas em prol do 
V Congresso Brasileiro de Informática na Educação (CBIE 2016)

Anais do XXVII Simpósio Brasileiro de Informática na Educação (SBIE 2016)

sucesso comum do grupo. Agente Professor - o Agente Professor (AProf) é responsável pela formação do agrupamento inicial de alunos, pelo planejamento e organização dos conteúdos de ensino e aprendizagem da disciplina e gestão de alunos. Agente AMPAR - o Agente de Mensuração de Perfil de AutoRrelatos (AMPAR) tem o objetivo de mensurar autorrelatos do aluno. O agente disponibiliza o TCLE (Termo de Consentimento Livre e Esclarecido) aos alunos. O aluno ao aceitar o termo, são coletados dados básicos sobre o mesmo, como nome, idade e sexo. Em seguida, ele é instruído a responder às três escalas de autorrelatos esperança, autoestima e autoeficácia. Ao responder as escalas, é mostrado ao aluno o seu perfil de autorrelato obtido, baseado na Tabela 1. Agente AMFAR: o Agente de Mensuração de Fitness de AutoRrelatos (AMFAR) é responsável pela tarefa de calcular o Potencial de Colaboração do Aluno ( $P C A$ ). Por definição, o PCA (fitness) é expresso por uma função matemática denominada função fitness ou função objetivo $\left(F_{\text {fitness }}\right)$ definida da forma que segue na Equação (1).

$$
P C A k \triangleq \sum_{i=1}^{3} a k i=a k 1+a k 2+a k 3 \quad \text { Equação (1) }
$$

onde,

$$
a k i=\left\{\begin{array}{c}
1, \leftrightarrow \text { se o PerfilautorrelatoAluno for } B \\
2, \leftrightarrow s \text { se o PerfilAutorrelatoAluno for } M B \\
3, \leftrightarrow \text { se o PerfiliutorrelatoAluno for } M \\
4, \leftrightarrow \text { se o PerfilAutorrelatoAluno for } M A \\
5, \leftrightarrow \text { se o PerfilAulorreluluAluru for } A
\end{array}\right\}
$$

com

$$
3 \leq P C A_{k} \leq 15 \text { e } 1 \leq a_{k i} \leq 5
$$

Agente AACG: o Agente Avaliador de Colaboração de Grupo (AACG) é responsável em calcular o Potencial de Colaboração do Grupo ( $P C G j)$. Por definição, para o $j$-ésimo grupo, o $P C G$ é calculado como na Equação (2).

$$
P C G j \triangleq \frac{1}{m j}[(\sqrt{(A+B+C)})] \quad \text { Equação (2) }
$$

onde,

$$
A=\left[\left(\sum_{k=1}^{n j} a j k 1\right) x\left(\sum_{k=1}^{n j} a j k 1\right)\right] \quad B=\left[\left(\sum_{k=1}^{n j} a j k 2\right) x\left(\sum_{k=1}^{n j} a j k 2\right)\right] \quad C=\left[\left(\sum_{k=1}^{n j} a j k 3\right) x\left(\sum_{k 3=1}^{n j} a j k 3\right)\right]
$$

Já o Fitness Global dos Grupos ( $F G G)$, por definição, é calculado como na Equação (3).

$$
F G G \triangleq \sum_{j=1}^{n j} P C G j \quad \text { Equação (3) }
$$

onde, $n_{g}$ é o número de grupos, com $3 \mathrm{n}_{\mathrm{g}} \leq F G G \leq 15 \mathrm{n}_{\mathrm{g}}$

Para inferir o nível de colaboração de grupo, o agente AACG usa logs do AVEA em conjunto com o componente de mineração de dados (regras de associação), de forma 
V Congresso Brasileiro de Informática na Educação (CBIE 2016)

Anais do XXVII Simpósio Brasileiro de Informática na Educação (SBIE 2016)

a descobrir padrões de comportamentos colaborativos. Bases de Dados: as Bases de Dados (BD) do SMA-Hermes são as Base de Dados de Perfis de Autorrelatos do Aluno (BDPAA), Base de Dados de Fitness de Autorrelatos do Aluno (BDFAA), Base de Dados de Desempenho de Colaboração de Grupo (BDDCG) e Logs do AVEA (LAVEA). Componente de Mineração de Dados: o Componente de Mineração de Dados (CMD) utilizado é Regra de Associação. Segundo [Fayyad et al. 1996], regras de associação são algoritmos que têm por objetivo encontrar relações entre dados que ocorrem com determinada frequência e que possam ser utilizados para identificar padrões de comportamentos. O CMD permite identificar se o perfil inicial do aluno definido inicialmente através de autorelato se mantém ou se modifica durante o processo de aprendizagem. Em caso de modificação, é necessário redefinir novos grupos de aprendizagem com objetivo de otimizar a aprendizagem colaborativa de cada grupo.

A modelagem do SMA-Hermes foi pautada no diagrama da estrutura global do sistema (Figura 2). O diagrama apresenta uma visão geral de todos os agentes envolvidos no suporte à formação otimizada de grupos. A modelagem completa do SMA-Hermes foi desenvolvida utilizando a metodologia orientada a agentes Prometheus [Padgham and Winikoff 2004] e a ferramenta PDT (do inglês Prometheus Design Tool).

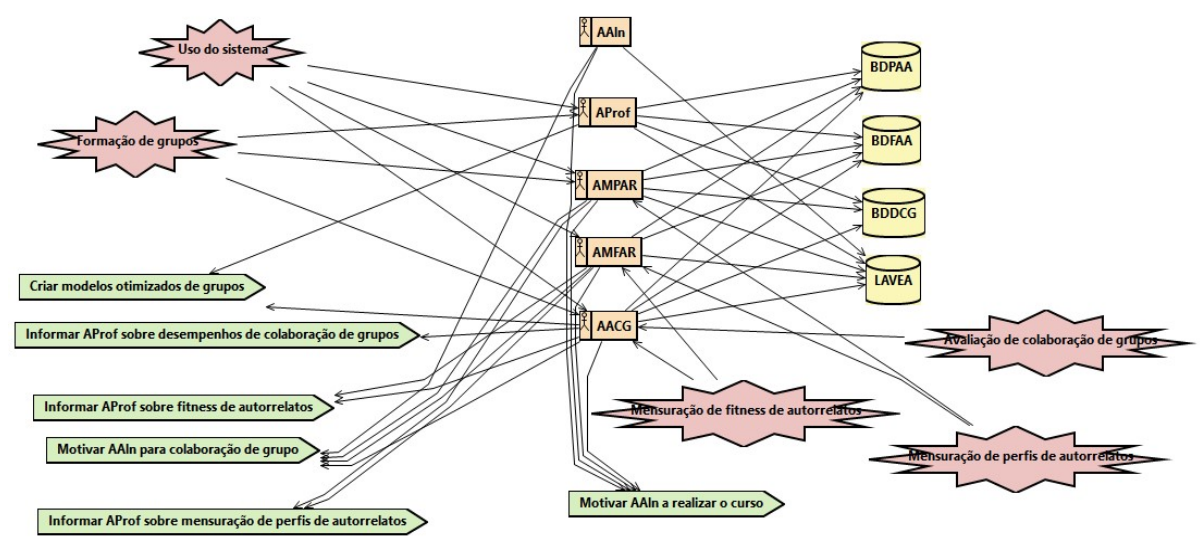

Figura 2: Diagrama da estrutura global do SMA-HERMES.

Fonte: Autor (2016).

Na Tabela 2 são descritos os componentes da estrutura global do SMA-Hermes. A notação simbólica usada é baseada na metodologia Prometheus [Pontes 2010].

Tabela 2: Descrição dos componentes do diagrama global do SMA-Hermes Fonte: Autor (2016).

\begin{tabular}{|c|c|c|}
\hline Legenda & Descrição & Exemplo do SMA-Hermes \\
\hline$\sum$ Perceptio & $\begin{array}{l}\text { Entradas perceptivas do agente em qualquer } \\
\text { momento dado [Russell and Norvig 2013]. }\end{array}$ & $\begin{array}{l}\text { uso do sistema, formação de grupos, mensuração de perfis de } \\
\text { autorrelatos, mensuração de fitness de autorrelatos e } \\
\text { avaliação de colaboração de grupos. }\end{array}$ \\
\hline Bases de Dados & $\begin{array}{l}\text { São as fontes de dados geradas usadas na } \\
\text { arquitetura do sistema [Pontes 2010]. }\end{array}$ & BDPAA, BDFAA, BDDCG e LAVEA. \\
\hline \begin{tabular}{|l|l|} 
Agente \\
\end{tabular} & $\begin{array}{l}\text { Entidade capaz de perceber um ambiente através } \\
\text { de sensores e atuar sobre o mesmo através de } \\
\text { atuadores [Russell and Norvig 2013]. }\end{array}$ & AAln, AProf, AMPAR, AMFAR e AACG. \\
\hline & $\begin{array}{l}\text { São tarefas que o agente é capaz de realizar. Elas } \\
\text { podem ser externas, quando alteram o ambiente, } \\
\text { ou internas, quando alteram somente as crenças } \\
\text { do agente, sem afetar o ambiente [Russell and } \\
\text { Norvig 2013]. }\end{array}$ & $\begin{array}{l}\text { informar AProf sobre mensurações de perfis de autorrelatos, } \\
\text { informar AProf sobre fitness de autorrelatos, informar AProf } \\
\text { sobre desempenhos de colaboração de grupos, criar modelos } \\
\text { otimizados de grupos, motivar AAln para colaboração de } \\
\text { grupo. }\end{array}$ \\
\hline
\end{tabular}


V Congresso Brasileiro de Informática na Educação (CBIE 2016)

Anais do XXVII Simpósio Brasileiro de Informática na Educação (SBIE 2016)

Na modelagem do SMA-Hermes, um protocolo de interação entre os agentes é especificado: protocolo de formação otimizada de grupos. Com o protocolo, o processo de formação de grupos envolve todos os agentes do SMA-Hermes. Este protocolo foi especificado utilizando a ferramenta Astah Professional, conforme ilustra a Figura 3.

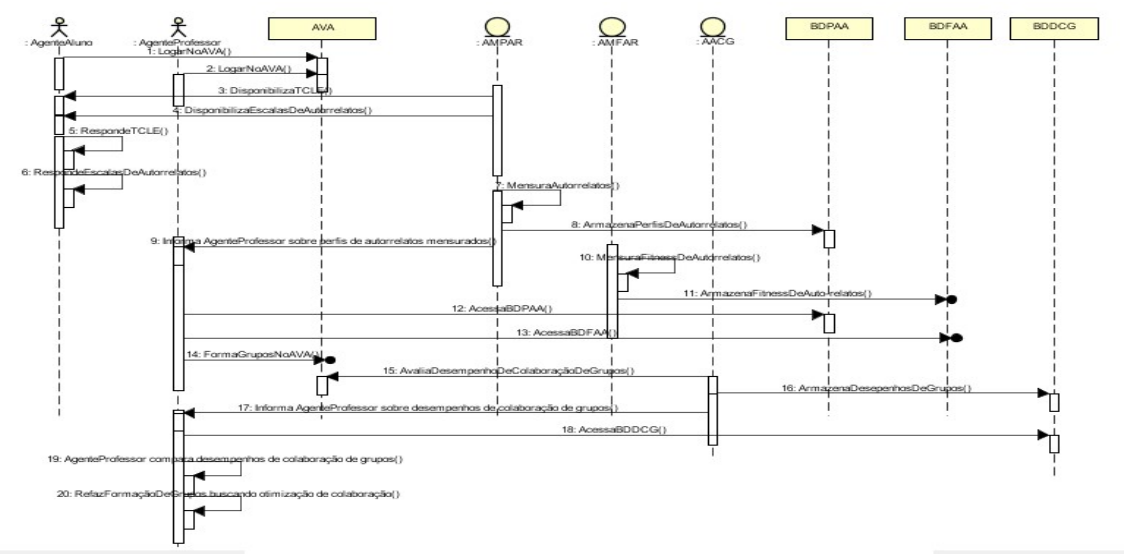

Figura 3: Protocolo de Formação de Grupos do SMA-Hermes.

Fonte: Autor (2016).

Da Figura 3, observa-se, que após o login dos agentes AAln e AProf no AVEA, o AMPAR disponibiliza ao aluno o TCLE. Efetivada esta etapa, os perfis de autorrelatos dos alunos são calculados pelo AMPAR, e armazenados na base de dados BDPAA à disposição do AProf para a formação do agrupamento inicial de alunos. A partir deste ponto, os agentes AMFAR e AACG são encarregados de calcularem, respectivamente, o $P C A$ e o $P C G j$, armazenando seus resultados nas respectivas bases de dados de fitness à disposição do AProf e do AACG.

\section{Metodologia de pesquisa}

Experimentos foram realizados no AVEA MOODLE/CINTED/UFRGS. Como passos metodológicos, adotou-se os que seguem: (a) Definição da amostra: A amostra foi composta de 36 alunos (faixa etária média de 18 anos), do curso de Eng Comp/UEMA, da disciplina de Matemática Discreta; (b) Mensuração dos perfis de autorrelatos: Usando o agente AMPAR, foram calculados os perfis de autorrelatos e armazenados na BDPAA à disposição dos agentes AProf e AMFAR; (c) Preparação da sala virtual: A sala virtual foi criada no MOODLE. Na sala, disponibilizou-se as atividades de aprendizagem. As ferramentas de apoio na realização das atividades foram chat, fórum e e-mail; (d) Formação de grupos: Para as composições dos grupos, definiu-se os critérios: (i) formação aleatória e (ii) formação pelo sistema. Para a formação (i), os grupos de alunos foram formados por sorteio. $\mathrm{Na}$ formação (ii) os alunos foram distribuídos da seguinte forma: $\operatorname{Grp}_{1}=\{$ autorrelatos B $\}, G r p_{2}=$ $\{$ autorrelatos $\mathrm{M}\}, \mathrm{Grp}_{3}=\{$ autorrelatos $\mathrm{A}\}$ e $\mathrm{Grp}_{4}=\{$ autorrelatos mesclados $\}$; (e) Atividades de aprendizagem: Foi definido que os grupos deveriam produzir um texto da disciplina, abrangendo conteúdos de lógica formal. O texto final construído pelos grupos devia conter: (i) introdução, (ii) desenvolvimento, (iii) conclusões) e (iv) referências. (f) Avaliação da aprendizagem: Variáveis como PMOA (Pontuação Máxima Obtida na Atividade), TRA (Tempo de Realização da Atividade) foram estabelecidas para avaliar a colaboração de grupo. Ao final dos experimentos foi aplicado um teste sociométrico, o mesmo adotado por [Quarto 2006]: a) Com quem você mais gostou de trabalhar em grupo e por quê? e b) Com quem você menos gostou de 
V Congresso Brasileiro de Informática na Educação (CBIE 2016)

Anais do XXVII Simpósio Brasileiro de Informática na Educação (SBIE 2016)

trabalhar em grupo e por quê? O objetivo do teste é encontrar afinidades sociais em função dos perfis de autorrelatos dos alunos e assim poder subsidiar a construção de uma ontologia de colaboração. A amostra de 36 alunos foi disposta em forma matricial quadrada de ordem 36 (Aln1, Aln2, Aln3, ... Aln36)x(Aln1, Aln2, Aln3, .., Aln36), com cada elemento desta matriz Aij representado por 1 quando um aluno expressava que gostou de trabalhar com outro colega, e 0, caso contrário. (g) Resultados: Os resultados são organizados e apresentados em forma de tabelas, gráficos e textos.

Tabela 3: Desempenho de grupo - Experimento 1.

\begin{tabular}{|c|c|c|c|c|c|c|c|}
\hline & \multicolumn{4}{|c|}{ MÉDIA } & \multirow[b]{2}{*}{ PCGj } & \multirow[b]{2}{*}{ PMOA } & \multirow[b]{2}{*}{ TRA } \\
\hline GRUPOS & Esperança & Autoestima & Autoeficácia & Autorrelato do grupo (ARG) & & & \\
\hline 3 & 3,3 & 4,8 & 3,8 & 3,92 & 6,9 & 7,5 & 2,0 \\
\hline 1 & 3,6 & 3,2 & 3,0 & 3,27 & 5,7 & 7,0 & 3,0 \\
\hline 5 & 3,4 & 3,0 & 2,8 & 3,07 & 5,3 & 3,5 & 1,0 \\
\hline 6 & 2,3 & 3,5 & 3,0 & 2,92 & 5,1 & 6,5 & 3,0 \\
\hline 2 & 2,3 & 3,5 & 2,8 & 2,83 & 5,0 & 2,0 & 3,0 \\
\hline 4 & 2,3 & 2,3 & 1,8 & 2,08 & 3,6 & 4,5 & 1,0 \\
\hline
\end{tabular}

Tabela 4: Desempenho de grupo - Experimento 2.

\begin{tabular}{|c|c|c|c|c|c|c|c|}
\hline & \multicolumn{5}{|c|}{ MÉDIA } & & \\
\hline GRUPOS & Esperança & Autoestima & Autoeficácia & ARG & PCGj & PMOA & TRA \\
\hline C & 3,75 & 4,50 & 5,00 & 4,42 & 7,70 & 9,50 & 1,00 \\
\hline A & 5,00 & 3,75 & 4,25 & 4,33 & 7,56 & 9,00 & 2,00 \\
\hline B & 2,00 & 5,00 & 3,75 & 3,58 & 6,56 & 8,00 & 2,00 \\
\hline F & 3,00 & 4,00 & 2,00 & 3,00 & 5,39 & 8,50 & 1,00 \\
\hline H & 2,00 & 3,50 & 3,00 & 2,83 & 5,02 & 10,00 & 1,00 \\
\hline I & 3,00 & 3,00 & 2,50 & 2,83 & 4,92 & 9,00 & 2,00 \\
\hline D & 1,75 & 2,75 & 2,50 & 2,33 & 4,11 & 6,00 & 1,00 \\
\hline E & 2,50 & 1,25 & 2,00 & 1,92 & 3,44 & 9,50 & 1,00 \\
\hline G & 2,00 & 2,25 & 1,00 & 1,75 & 3,17 & 9,00 & 1,00 \\
\hline
\end{tabular}

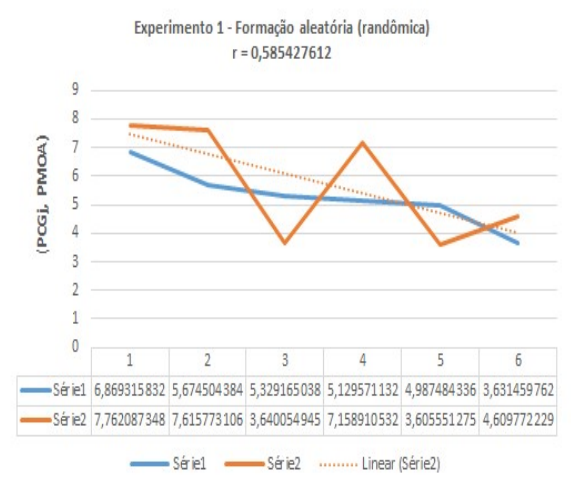

(a)

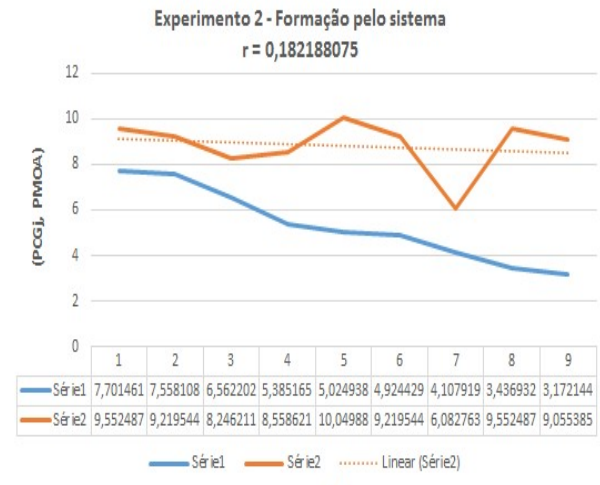

(b)

Figura 4: a) e b), respectivamente previsões lineares dos experimentos 1 e 2. Fonte: Autor (2016). 
V Congresso Brasileiro de Informática na Educação (CBIE 2016)

Anais do XXVII Simpósio Brasileiro de Informática na Educação (SBIE 2016)

Em razão do tamanho da matriz 36x36, apresenta-se apenas parte da mesma (Tabela 5), assim como na Tabela 6, parte das representações de "1s" e " 0 " entre os pares, ou seja, seus perfis de autorrelatos e suas respectivas características expressas pelos seus pares de grupo.

Tabela 5: Resultado parcial do teste sociométrico.

\begin{tabular}{|c|c|c|c|c|c|c|c|c|}
\hline \multirow{3}{*}{ Tuplas } & \multicolumn{3}{|c|}{ Aluno $x$} & \multicolumn{3}{|c|}{ Aluno y } & \multirow{3}{*}{ Afinidades } & \multirow{3}{*}{ Por quê? } \\
\hline & \multicolumn{3}{|c|}{$\begin{array}{c}\text { Perfil de } \\
\text { Autorrelatos }\end{array}$} & \multicolumn{3}{|c|}{$\begin{array}{c}\text { Perfil de } \\
\text { Autorrelatos }\end{array}$} & & \\
\hline & \multicolumn{3}{|c|}{ esp/aes/aef } & \multicolumn{3}{|c|}{ esp/aes/aef } & & \\
\hline $\begin{array}{l}\text { (Aln1, } \\
\text { Aln2) }\end{array}$ & M & B & B & A & MA & MA & 1 & $\begin{array}{l}\mathrm{O} \text { Aln2 possui uma linha de } \\
\text { raciocínio bem parecida com a minha } \\
\text { e isso facilita as coisas. }\end{array}$ \\
\hline $\begin{array}{l}(A \ln 15, \\
\text { Aln26) }\end{array}$ & A & A & A & MA & MB & MA & $\mathbf{0}$ & $\begin{array}{l}\text { O Aln26 se distraía muito e nunca } \\
\text { estava muito aberto às ideias } \\
\text { coletivas. }\end{array}$ \\
\hline
\end{tabular}

Tabela 6: Representações de "1s" das respostas do teste sociométrico.

\begin{tabular}{|c|l|}
\hline $\begin{array}{c}\text { Perfil de Autorrelatos do } \\
\text { Aluno y }\end{array}$ & \multicolumn{1}{c|}{ Características do Aluno y expressas pelo seu par Aluno x } \\
\hline esp: MB; aes: $\mathrm{M} ;$ aef: $\mathrm{M}\}$ & Se esforça bastante. Ajuda o grupo nas atividades. \\
\hline esp: $\mathrm{A} ;$ aes: $\mathrm{B} ;$ aef: $\mathrm{M}\}$ & $\begin{array}{l}\text { É focado em responder as atividades de aprendizagem. Ajuda o grupo naquilo que } \\
\text { entende sobre o assunto aprendido. Coopera para o bom andamento do grupo. }\end{array}$ \\
\hline esp: MA; aes: A; aef: $\mathrm{A}\}$ & Ajuda os outros membros do grupo a resolver suas atividades de aprendizagem. \\
\hline esp: A; aes: A; aef: A $\}$ & $\begin{array}{l}\text { Demonstra maior comprometimento com a resolução das atividades de aprendizagem. } \\
\text { Motiva os membros do grupo com foco no sucesso. Promove interações interpessoais } \\
\text { no grupo. Ajuda os colegas de grupo. Seriedade e compromisso com o trabalho de } \\
\text { grupo e organização. Equilibra diferenças no grupo. }\end{array}$ \\
\hline
\end{tabular}

\section{Análise e discussões}

Baseado nos resultados, verificou-se que a variável TRA não ofereceu contribuição para a análise de desempenho de grupo. Já a variável $P C G j$ ofereceu resultados positivos, apresentando uma relevante tendência/correlação com $P M O A$. Verificou-se que, em ambos os experimentos, a previsão linear mostra que quanto maior o valor de $P C G j$ maior será a qualidade no grupo, isto é, maior valor para $P M O A$. Conclui-se com isso que a variável $P C G j$ é um indicador de colaboração de grupo baseado em perfis de autorrelatos esperança, autoestima e autoeficácia.

Constatou-se que os melhores desempenhos de grupo são encontrados nos grupos cujos perfis de autorrelatos são de médio para alto e os mais baixos se encontram naqueles grupos com os perfis de autorrelatos são baixos. Notou-se, também, que os melhores desempenhos de grupos são manifestados em formações de grupos heterogêneas, ocorrendo esse fato nos dois experimentos. Do teste sociométrico, podese extrair características ponderantes dos pares afins, expressas através das respostas deste, como: "se esforça em..."; "ajuda o grupo a..."; "se importa com..."; "é focado em..."; "coopera para..."; "tem paciência com..."discute os conflitos"; "aceita opiniões 
V Congresso Brasileiro de Informática na Educação (CBIE 2016)

Anais do XXVII Simpósio Brasileiro de Informática na Educação (SBIE 2016)

diversas..."; "sabe trabalhar colaborativamente..."; "demonstra comprometimento..."; "motiva o grupo..."; "demonstra seriedade e compromisso..."; "equilibra diferenças no grupo..."; "se distrai muito e nunca estava muito aberto às ideias coletivas".

Acredita-se que essas expressões afetivas entre os pares úteis na construção de uma ontologia de colaboração, e assim poder auxiliar a modelagem de papéis do aluno baseados em seus perfis de autorrelatos, bem como na construção de regras de associação do tipo $p_{1} \rightarrow p_{2}$, onde $p_{1}$ representaria o perfil de autorrelatos do aluno e $p_{2} \mathrm{o}$ seu papel associado a esse perfil.

\section{Conclusões e trabalhos futuros}

Este trabalho apresentou um modelo de tecnologia que considera perfis de autorrelatos de alunos no auxílio à formação otimizada de grupos para aprendizagem colaborativa. Os resultados se mostraram satisfatórios em relação à melhoria das performances de grupos quando se considera a variável $P C G j$. Como trabalhos futuros, vislumbra-se mais experimentos na investigação, principalmente envolvendo a comunicação de todos os componentes propostos na arquitetura do SMA-Hermes. A concepção e implementação da ontologia de colaboração baseada em perfis de autorrelatos esperança, autoestima e autoeficácia.

\section{Referências}

Bandura, A. (1997). Self-efficacy: The exercise of control. New York: W.H. Freeman.

Baumeister, R. F., et al. (2003) "Does high self-esteem cause better performance, interpersonal success, happiness, or healthier lifestyles?." Psychological science in the public interest 4.1 (2003): 1-44.

Bercht, M. Computação afetiva: vínculos com a psicologia e aplicações na educação. In: Psicologia \& Informática Produções do III PsicoInfo e II Jornada do NPPI, 1ํㅡ edição, 2006, p. 106-115. Disponível em: <http://newpsi.bvspsi.org.br/ebooks2010/en/Acervo_files/PsiInfo.pdf $>$ Acesso em 16 de Jan 2015.

Bressler, L. et al. (2011). "Demographic and psychographic variables and the effect on online student success." Journal of Technology Research 2 (2011): 1-16.

Cunha, L. M. et al. (2002). Hugo Fuks, and Carlos José Pereira de Lucena. "Suporte a Grupos de Trabalho em Turmas do Ambiente AulaNet." Anais do Simpósio Brasileiro de Informática na Educação. Vol. 1. No. 1. 2002.

Fayyad, U. M. et al. (1996). From Data Mining to Knowledge Discovery: overview in Knowledge Discovery and Data Mining, AAAI, 1996.

Flick, Uwe. Introdução à metodologia de pesquisa: um guia para iniciantes. Porto Alegre: Penso, 2013.

Hanson, J. M. et al (2010) "Hope uniquely predicts objective academic achievement above intelligence, personality, and previous academic achievement." Journal of Research in Personality 44.4 (2010): 550-553.

Hutz, C. S. et al. (2014). A escala de autoestima de Rosenberg. In: Avaliação em Psicologia Positiva/Organizador, Cláudio Simon Hutz - Porto Alegre: Artmed, 2014.

Monteserin, A. et al. (2010) "Análisis de la formación de grupos en Aprendizaje Colaborativo Soportado por Computadoras." XXI Simpósio Brasileiro de Informática na Educação (SBIE). Brasil (2010). 
V Congresso Brasileiro de Informática na Educação (CBIE 2016)

Anais do XXVII Simpósio Brasileiro de Informática na Educação (SBIE 2016)

Pacico, J. C. et al. (2014). Autoeficácia - Yes We Can! In: Avaliação em Psicologia Positiva/Organizador, Cláudio Simon Hutz - Porto Alegre: Artmed, 2014.

Pacico, J. C. and Bastianello, M. R. Instrumentos para avaliação da esperança disposicional e escala de esperança cognitiva. In: Avaliação em Psicologia Positiva/Organizador, Cláudio Simon Hutz - Porto Alegre: Artmed, 2014.

Padgham, L. and Winikoff, M. Developing intelligent agent systems: a practical guide. [S.1.]: John Wiley and Sons, 2004.

Pontes, A. A. A. Uma arquitetura de agentes para suporte à colaboração na aprendizagem baseada em problemas em ambientes virtuais de aprendizagem. Diss. Universidade Federal Rural do Semi-Árido, 2010

Quarto, C. C.. "Inferindo Fatores Sócio-Afetivos em Ambientes de EnsinoAprendizagem Colaborativos Assistidos por Computador." (2006). Dissertação de Mestrado Acadêmico. Programa de Pós-Graduação em Engenharia de Eletricidade (PPGEE) - Universidade Federal do Maranhão (UFMA. São Luís - MA.

Reis, R. et al. (2015a). "Relação entre os Estados Afetivos e as Teorias de Aprendizagem na Formação de Grupos em Ambientes CSCL." Anais do Simpósio Brasileiro de Informática na Educação. Vol. 26. No. 1. 2015.

Russell, S. and Norvig, P. Artificial intelligence: A modern approach, 3a. ed. [S.1.]: Rio de Janeiro: Elsevier, 2013

Seligman, M. e Csikszentmihalyi, M. (2000). Positive psychology: An introduction. American Psychologist, 55, 5-14.

Snyder, C. R. et al. (2002). "Hopeful choices: A school counselor's guide to hope theory." Journal of Personality and Social Psychology 65 (2002): 1061-1070.

So, H. and Brush, T. "Student perceptions of collaborative learning, social presence and satisfaction in a blended learning environment: Relationships and critical factors." Computers \& Education 51.1 (2008): 318-336.

Stahl, G et al. (2006). Computer-supported collaborative learning: An historical perspective. In R. K. Sawyer (Ed.), Cambridge handbook of the learning sciences (pp. 409-426). Cambridge, UK: Cambridge University Press. Disponível em: $<$ http://GerryStahl.net/cscl/CSCL_English.pdf $>$ Acesso em: 14 de jun. de 2014.

Tierney, W. G. "Addressing Failure: Factors Affecting Native American College Student Retention." Journal of Navajo Education 13.1 (1995): 3-7.

Weiss, G. Multiagent systems: a modern approach to distributed artificial intelligence. MIT press, 1999.

Wooldridge, M. J. An Introduction to Multiagent Systems. [S.1.]: John Wiley \& Sons, 2008. 